\title{
Comparaison de l'accès au premier emploi des apprentis et des lycéens
}

\author{
Liliane BONNAL* Sylvie MENDES** \\ Catherine SOFER *
}

\begin{abstract}
RÉSUMÉ. - Notre but est de comparer l'accès au premier emploi des apprentis et des lycéens et par conséquent de mesurer l'effet «pur " d'une formation en entreprise. Le modèle utilisé estime les paramètres associés aux probabilités d'être apprenti et d'être en emploi ainsi que ceux associés à la fonction de densité de la durée de la période de chômage observée. Les apprentis semblent avoir un avantage sur les lycéens, en terme d'accès au premier emploi.
\end{abstract}

\section{Access to the first job : Apprenticeship versus vocational shool}

ABSTRACT. - Our aim is to compare the access to the first job of apprentices and vocational school leavers and therefore measure the «pure » within-firm training effect. The model estimates the parameters linked to the probabilities of being an apprentice and being employed, and those associated to the probability density function of the unemployment duration. Apprentices seem to have a distinct advantage over those who attended vocational school in term of access to the first job.

\footnotetext{
* Gremaq et Leerna, Université des Sciences Sociales de Toulouse, 21 allée de Brienne, Bât. F, 31000 Toulouse. Tél : 05611285 43, Email : bonnal@ cict.fr

${ }^{* \star}$ Léo-Cereq, Faculté de Droit, d'Économie et de Gestion, rue de Blois, BP 6739, 45067 Orléans cedex 2.Tél : 02384173 65, Email : sylvie.mendes@univ-orleans.fr.

- Team, Université Paris I-Panthéon-Sorbonne, Maison des Sciences Économiques, 106-112 Bd de l'Hôpital, 75647 Paris cedex 13. Tél : 33 (0) 1440782 58, Fax : 33 (0) 1440782 47, Email : Catherine.Sofer@univ-paris1.fr
}

JEL Classification : J 21, J 24.

Mots clé : transition de l'école à l'emploi, apprentissage, chômage des jeunes, marché du travail des jeunes 


\section{Introduction}

La formation en entreprise est-elle la source de compétences plus valorisées par l'entreprise que le savoir acquis à l'école ${ }^{1}$ ? De nombreuses politiques, notamment celles visant à réduire le chômage des jeunes, ont été récemment élaborées en France pour faciliter et accroître le temps passé en emploi dans les entreprises grâce à des programmes scolaires ou de formation ${ }^{2}$. Le développement de l'apprentissage, qui s'est fortement accru ces dernières années, est l'une de ces politiques ${ }^{3}$. La question est donc de savoir si cette forme particulière d'enseignement facilite la transition de l'école à l'emploi, comme semble le montrer l'exemple allemand, ou non. Le faible taux de chômage des jeunes en Allemagne (aujourd'hui, nous devrions plutôt parler du relativement faible taux de chômage des jeunes) a traditionnellement été attribué principalement au développement de l'apprentissage dans ce pays. Mais il pourrait exister d'autres explications, telles qu'une spécificité démographique, ou des arrangements institutionnels augmentant les revenus du capital humain spécifique et/ou général pour l'entreprise, ou plus généralement les bénéfices de l'emploi des jeunes, par exemple.

Notre but est ici d'essayer de mesurer l'effet de la formation en entreprise en comparant la transition de l'école à l'emploi des apprentis avec celle des jeunes ayant atteint le même niveau, mais dans un lycée professionnel, i.e. dont l'enseignement tant théorique que pratique se fait principalement au lycée. Nous considérons ici les jeunes ayant préparé un CAP, soit par la voie de l'apprentissage soit par le lycée professionnel. Ce diplôme correspond au premier niveau de sortie du lycée professionnel (environ douze ans d'études à partir de l'âge de scolarité obligatoire) (niveau V).

Notre étude devrait permettre de répondre à un certain nombre de questions, par exemple de savoir s'il est nécessaire de maintenir un système permettant d'atteindre un même niveau de formation par deux voies distinctes : l'apprentissage et le lycée professionnel. Ou encore, si la description de la trajectoire scolaire s'arrête au niveau de formation atteint ou bien si le mode de formation est également un indicateur de cette trajectoire (RYAN [2001]). Certaines études, telles que celles de AdDison et Siebert [1994], Booth et SATChell [1994], Mansuy [1996], Vialla [1997], et Bonnal, Fleury et Rochard [1999], se sont donc attachées à décrire le parcours des jeunes passés par l'apprentissage.

Notre objectif dans ce qui suit est de comparer l'accès au premier emploi des apprentis et des lycéens et, en particulier, les durées de chômage avant l'obtention du premier emploi.

Notre travail s'inscrit donc dans l'ensemble des travaux sur le chômage des jeunes de bas niveau scolaire ayant préparé leur diplôme soit par apprentissage soit dans un lycée professionnel (par exemple LYNCH [1983], TORELLI et Trivellato [1989], Dolton, Makepeace et Treble [1994], Bonnal, Mendes et Sofer [2002], Sollogoub et UlRich [1999]).

\footnotetext{
1. Nous tenons à remercier ici les rapporteurs anonymes de la revue pour leurs commentaires et leurs suggestions. Les auteurs sont, bien entendu, seuls responsables des erreurs qui pourraient subsister.

2. «Un lycée pour $\mathrm{XXI}^{\mathrm{e}}$ siècle - L'enseignement professionnel intégré » : conférence de presse du 24 juin 1999 de Claude ALLÈGRE.

3. Voir Liaisons sociales, 1990, 1993.
} 
Nos principaux apports sont les suivants :

Pour mesurer l'effet du passage par l'apprentissage sur l'accès au premier emploi :

- nous tenons compte du caractère éventuellement endogène du passage par l'apprentissage. En effet, les jeunes qui choisissent l'apprentissage plutôt que le lycée professionnel pourraient présenter certaines caractéristiques exerçant une influence directe sur leur probabilité de choix,

- nous nous interrogeons sur le rôle du capital humain spécifique et nous nous demandons, d'une part si les apprentis ont plutôt tendance à s'insérer chez leur maître d'apprentissage que dans une autre entreprise, et d'autre part si ceux qui ne l'ont pas fait sont plutôt favorisés ou défavorisés par rapport aux lycéens.

Le modèle considéré dans ce travail va donc nous permettre de mesurer simultanément les probabilités :

- de préparer son diplôme par l'apprentissage,

- de trouver un emploi immédiatement après la sortie du système éducatif,

- d'être embauché chez son maître d'apprentissage (cette probabilité ne concerne que les individus apprenti(e)s),

- d'étudier la durée moyenne de chômage des jeunes n'ayant pas trouvé un emploi immédiatement après la sortie du système éducatif.

Les estimations seront menées à partir de l'enquête «Panel mesures jeunes » du Céreq ${ }^{4}$, qui nous renseigne sur les différents états occupés sur le marché du travail (emploi, chômage, inactivité) au cours des 18 mois qui suivent la sortie du système éducatif. Nous nous concentrerons ici sur l'accès au premier emploi.

D'après les résultats obtenus, il semblerait que le passage par l'apprentissage ait un effet positif sur la probabilité de trouver immédiatement un emploi.

Pour les hommes comme pour les femmes, la probabilité de sortie immédiate est plus forte pour les apprentis que pour les lycéens. D'autre part, pour ceux qui ne restent pas chez leur maître d'apprentissage, on observe des différences entre les filles et les garçons. Sur la population masculine, les apprentis diplômés ont la même probabilité que les lycéens de trouver un emploi (75\%). En revanche, pour les non diplômés, il semblerait que les apprentis conservent un avantage dans l'obtention d'un emploi immédiat (64\% des «movers » contre $59.5 \%$ des lycéens trouvent un emploi immédiatement). Les entreprises ne semblent donc pas considérer que le lycée professionnel délivre une meilleure qualité ou quantité de capital humain général que l'apprentissage. La situation opposée apparaît chez les femmes, pour lesquelles, mêmes si dans l'ensemble les apprenties réussissent mieux, les « movers » font moins bien $(47 \%$ des « movers », contre $58.4 \%$ des lycéennes diplômées et $38.6 \%$ des «movers » contre $45.6 \%$ des lycéennes non diplômées, trouvent un emploi immédiatement).

Nous présenterons dans la section 2 les données et quelques statistiques descriptives. La section 3 sera consacrée à la présentation du modèle, et la section 4 aux résultats. Nous conclurons enfin notre analyse dans la section 5 .

4. Centre d'Études et de Recherches sur les Qualifications. 


\section{Les données}

Les données proviennent de l'enquête «Panel mesures jeunes » du Céreq. Pour permettre une évaluation des mesures jeunes, le Céreq a procédé, à partir de 1986, à trois enquêtes téléphoniques auprès de jeunes n'ayant pas le baccalauréat général (WERQUIN [1997]). Il semble en effet que les dispositifs d'aide à l'emploi des jeunes étaient initialement plutôt destinés aux jeunes de niveau inférieur à celui du baccalauréat général.

Nous considérons le deuxième panel téléphonique du Céreq, comportant cinq vagues, qui interroge 2500 jeunes représentatifs de la cohorte des jeunes sortis en 1989 de l'enseignement secondaire ou d'un centre de formation d'apprentis (CFA). «Ce groupe est considéré comme représentatif de l'ensemble des jeunes (396 372) sortis aux niveaux considérés en 1989 » (WERQUIN [1996]).

Nous utilisons ici la première vague de cette enquête, soit une observation des jeunes sur les 18 mois qui suivent leur sortie, entre juin 1989 et décembre 1990. Notre travail porte sur les jeunes ayant préparé un CAP ou un BEP par apprentissage ou par voie scolaire. Notre échantillon de travail est ici de 1399 observations.

Les statistiques qui vont être présentées par la suite ont été pondérées afin qu'elles soient représentatives. Comme pour la plupart des études sur le sujet, deux sous populations ont été considérées : la population des hommes et la population des femmes. La raison principale est, comme nous le verrons dans le tableau 1, que les spécialités préparées par les jeunes ne sont pas, selon le sexe, les mêmes 5 .

Les principales statistiques descriptives sont données dans le tableau 1. Cet échantillon compte plus d'hommes que de femmes $(58.7 \%)$. Le pourcentage d'apprentis est de l'ordre de $48.2 \%$ (36.1\% pour la population féminine et $56.7 \%$ pour la population masculine). Plus de $60 \%$ des individus ont obtenu leur diplôme. Ce pourcentage est légèrement plus élevé pour les femmes et les apprentis (68\% des apprenties contre $61 \%$ des apprentis et $62.4 \%$ des lycéennes contre $59 \%$ des lycéens). $82.5 \%$ des diplômes préparés par la population féminine sont liés au secteur tertiaire. Les hommes s'orientent principalement vers les métiers du bâtiment, de l'électricité, de la mécanique, de l'alimentation et dans une moindre mesure de la gestion et de l'hôtellerie. Les femmes, quant à elles, s'intéressent plutôt aux métiers liés à la gestion et au secrétariat, à l'esthétique, à l'hôtellerie et à la confection. $20 \%$ des jeunes ont un père à la recherche d'un emploi (tableau 1A dans l'annexe 1), ce pourcentage est plus élevé pour les lycéen(ne)s. Plus de $80 \%$ d'entre eux ont un père ouvrier ou employé, le pourcentage étant une fois encore plus élevé pour les lycéen(ne)s.

Le tableau 2 nous donne des informations sur la situation des jeunes sur le marché du travail.

5. Il existe de nombreux travaux observant une disparité entre les spécialités de formation pour les diplômes de niveau V selon le sexe. On peut citer par exemple BONNAL, FLeURY et ROCHARD [1999] ou Bonnal et MENDEs [2000]. 
TABLEAU 1

Statistiques descriptives (en pourcentage)

\begin{tabular}{|c|c|c|c|c|c|c|}
\hline & \multicolumn{3}{|c|}{ Hommes } & \multicolumn{3}{|c|}{ Femmes } \\
\hline Variables & Ens. $\mathrm{H}$ & Apprenti & Lycéen & Ens. F & Apprentie & Lycéenne \\
\hline Effectif total & 58.7 & 56.7 & 43.3 & 41.3 & 36.1 & 63.9 \\
\hline Les jeunes ont & & & & & & \\
\hline - obtenu le BEPC & 18.5 & 8.5 & 31.4 & 27.2 & 14.0 & 34.7 \\
\hline - préparé un BEP & 9.5 & 0.0 & 21.8 & 22.6 & 0.0 & 35.4 \\
\hline $\begin{array}{l}\text { - préparé leur diplôme } \\
\text { par apprentissage }\end{array}$ & 56.7 & 100.0 & 0.0 & 36.1 & 100.0 & 0.0 \\
\hline - obtenu leur diplôme & 60.1 & 61.1 & 58.8 & 64.4 & 67.9 & 62.4 \\
\hline $\begin{array}{l}\text { - trouvé un emploi à la } \\
\text { sortie du syst éducatif }\end{array}$ & 76.1 & 82.4 & 67.8 & 58.1 & 66.8 & 53.1 \\
\hline Spécialité du diplôme & & & & & & \\
\hline - secondaire & 64.7 & 60.8 & 69.8 & 17.5 & 9.0 & 22.2 \\
\hline - tertiaire & 35.3 & 39.2 & 30.2 & 82.5 & 91.0 & 77.8 \\
\hline - métier du bâtiment & 25.6 & 33.4 & 15.5 & & & \\
\hline - électricité, mécanique & 30.1 & 17.4 & 46.7 & & & \\
\hline - alimentation & 16.8 & 27.1 & 3.4 & 2.4 & 4.5 & 1.1 \\
\hline - gestion, secrétariat & 7.2 & 4.6 & 10.5 & 49.5 & 50.0 & 49.3 \\
\hline - hôtellerie & 3.3 & 4.3 & 2.1 & 17.5 & 14.9 & 19.0 \\
\hline - textile & & & & 13.0 & 3.4 & 18.5 \\
\hline - métier lié à l'esthétique & & & & 10.6 & 19.1 & 5.9 \\
\hline - autre spécialité & 17.0 & 13.2 & 21.8 & 7.0 & 8.1 & 6.2 \\
\hline $\begin{array}{l}\text { Âge moyen à la sortie du } \\
\text { système éducatif (en années) }\end{array}$ & 18.7 & 18.7 & 18.7 & 18.8 & 18.8 & 18.8 \\
\hline
\end{tabular}

Le pourcentage des jeunes hommes n'ayant pas connu de période de chômage est plus élevé que celui des femmes (76\% contre $58 \%$ ). De plus, parmi les apprenti(e)s, $82 \%$ des hommes et $67 \%$ des femmes ont trouvé un emploi immédiatement ${ }^{6}$ tandis que parmi les lycéen(ne)s on en observe $68 \%$ chez les hommes et $53 \%$ chez les femmes. Excepté pour les lycéens le diplôme favorise l'accès direct à un emploi. La moitié des apprenti(e)s ayant trouvé un emploi à la sortie du système éducatif a été embauchée par l'entreprise d'apprentissage. Ce pourcentage est légèrement plus faible pour les jeunes n'ayant pas obtenu leur diplôme.

Parmi les jeunes ayant connu une période de chômage, la durée moyenne de chômage des hommes est de 6 mois (elle est légèrement plus élevée pour les apprentis) et de 8 mois pour les femmes (10 pour les apprenties et 7 pour les lycéennes). Excepté pour les apprenties, le fait de ne pas avoir obtenu son diplôme augmente la durée moyenne de chômage.

À la fin de la période d'observation (18 mois après la sortie du système éducatif), $7 \%$ des hommes (10\% pour les apprentis et $6 \%$ pour les lycéens) et $20 \%$ des femmes (23\% des apprenties et $18 \%$ pour les lycéennes) n'ont toujours pas trouvé d'emploi. $7 \%$ des femmes ( $4 \%$ des apprenties et $8 \%$ des lycéennes) ont quitté momentanément le marché du travail à l'issue de la période de chômage. Les hommes quittent principalement la situation de

6. Étant donné la date de sortie du système éducatif (juin-juillet), nous considérons qu'un emploi immédiat s'obtient lorsque la durée de chômage est inférieure ou égale à 2 mois. 
TABLEAU 2

Situation sur le marché du travail (chiffres en pourcentage)

\begin{tabular}{|c|c|c|c|c|c|c|}
\hline \multirow[b]{2}{*}{ Variables } & \multicolumn{3}{|c|}{ Hommes } & \multicolumn{3}{|c|}{ Femmes } \\
\hline & Total H & Apprenti & Lycéen & Total F & Apprentie & Lycéenne \\
\hline \multicolumn{7}{|l|}{$\begin{array}{l}\text { Emploi immédiat chez le } \\
\text { maître d'apprentissage }\end{array}$} \\
\hline - ensemble & 21.9 & 38.7 & - & 12.5 & 34.6 & - \\
\hline - diplômé & 25.1 & 43.6 & - & 14.8 & 38.9 & - \\
\hline - non diplômé & 17.2 & 31.1 & - & 8.2 & 25.4 & - \\
\hline \multicolumn{7}{|l|}{$\begin{array}{l}\text { Emploi immédiat (à la sortie } \\
\text { du système éducatif) }\end{array}$} \\
\hline - ensemble & 76.1 & 82.4 & 67.8 & 58.1 & 66.8 & 53.1 \\
\hline - diplômé & 80.0 & 88.9 & 68.0 & 62.3 & 72.2 & 56.1 \\
\hline - non diplômé & 70.1 & 72.1 & 67.6 & 50.5 & 55.3 & 48.1 \\
\hline \multicolumn{7}{|l|}{$\begin{array}{l}\text { Parmi les jeunes en emploi } \\
\text { immédiat } \\
\text { Embauché par son maître } \\
\text { d'apprentissage }\end{array}$} \\
\hline - ensemble & 28.9 & 47.0 & - & 21.5 & 51.8 & - \\
\hline - diplômé & 31.4 & 49.0 & - & 23.8 & 53.9 & - \\
\hline - non diplômé & 24.5 & 43.1 & - & 16.3 & 45.8 & - \\
\hline \multicolumn{7}{|l|}{$\begin{array}{l}\text { Parmi les jeunes en situation } \\
\text { de chomage } \\
\text { Durée moyenne de chômage } \\
\text { (écart-type) }\end{array}$} \\
\hline - ensemble & $\begin{array}{c}5.9 \\
(3.8)\end{array}$ & $\begin{array}{c}6.7 \\
(4.4)\end{array}$ & $\begin{array}{c}5.3 \\
(3.3)\end{array}$ & $\begin{array}{c}8.1 \\
(5.6)\end{array}$ & $\begin{array}{l}10.0 \\
(5.2)\end{array}$ & $\begin{array}{c}7.3 \\
(5.6)\end{array}$ \\
\hline - diplômé & $\begin{array}{c}5.2 \\
(3.2)\end{array}$ & $\begin{array}{c}5.5 \\
(3.8)\end{array}$ & $\begin{array}{c}5.0 \\
(2.9)\end{array}$ & $\begin{array}{c}7.8 \\
(5.4)\end{array}$ & $\begin{array}{l}10.0 \\
(5.0)\end{array}$ & $\begin{array}{c}6.9 \\
(5.3)\end{array}$ \\
\hline $\begin{array}{l}\text { - non diplômé } \\
\text { Issue de la période } \\
\text { de chômage }\end{array}$ & $\begin{array}{c}6.6 \\
(4.3)\end{array}$ & $\begin{array}{c}7.4 \\
(4.6)\end{array}$ & $\begin{array}{c}5.7 \\
(3.9)\end{array}$ & $\begin{array}{c}8.4 \\
(5.8)\end{array}$ & $\begin{array}{l}10.0 \\
(5.4)\end{array}$ & $\begin{array}{c}7.8 \\
(5.9)\end{array}$ \\
\hline - Durée censurée & 7.0 & 10.0 & 5.8 & 20.1 & 23.4 & 18.3 \\
\hline $\begin{array}{l}\text { - Emploi sur Contrat } \\
\text { de Durée Indéterminée }\end{array}$ & 12.3 & 14.0 & 11.6 & 14.7 & 19.8 & 11.9 \\
\hline $\begin{array}{l}\text { - Emploi sur Contrat } \\
\text { de Durée Indéterminée }\end{array}$ & 28.7 & 26.0 & 29.7 & 31.3 & 41.5 & 25.8 \\
\hline - Emploi aidé & 14.0 & 10.0 & 15.7 & 27.2 & 11.7 & 35.6 \\
\hline - Inactivité & 38.0 & 40.0 & 37.2 & 6.7 & 3.6 & 8.4 \\
\hline
\end{tabular}

chômage pour un emploi sur contrat à durée déterminée. Les emplois sur contrats à durée indéterminée sont plutôt signés par des apprentis tandis que les emplois aidés sont plutôt occupés par les lycéens. Les apprenties occupent plutôt des emplois sur contrat à durée déterminée et dans une moindre mesure sur contrat à durée indéterminée. Les lycéennes sont quant à elles majoritairement en emplois aidés et dans une moindre mesure en emploi sur contrat à durée déterminée 7 .

7. Dans ce travail, nous ne nous intéressons pas directement à l'hétérogénéité des issues de la durée du chômage. Pour une étude plus détaillée de ce problème sur ce même échantillon, $c f$. BonNAL et MENDES [2000]. 


\section{Le modèle}

Les variables relatives à l'apprentissage sont d'un grand intérêt pour nous. Comparé au lycée professionnel, l'apprentissage peut être associé à des caractéristiques différentes exerçant des effets opposés sur le marché du travail. Notre ambition n'est pas ici de développer un modèle théorique complet, qui tienne compte à la fois du choix de filière éducative (cf. WILLIS et RoSEN [1979], WILLIS [1986]), et du choix d'investissement en capital humain spécifique de la part des individus et des firmes ( $c f$. НАSнімото [1981], КАто [1989]), cf. également STEEDMAN [1993]). L'analyse empirique développée ci-dessous permettra de trancher entre différentes hypothèses :

- le choix d'une filière d'apprentissage correspond-il plutôt à un choix positif de filière de la part des collégiens (ou de leurs parents), la sélection s'opérant à partir du nombre de places disponibles dans les entreprises acceptant des apprentis, ou bien s'agit-il d'un choix subi (imposé ou fortement orienté par des enseignants qui refuseraient ces jeunes dans des filières plus valorisées). Dans le premier cas, on peut s'attendre à ce que les jeunes qui choisissent l'apprentissage aient plutôt des caractéristiques positives (vis-à-vis des capacités de réussite scolaire et d'insertion sur le marché du travail). Dans le second cas, au contraire, le «choix » de l'apprentissage serait associé à des caractéristiques négatives.

- Théoriquement, l'apprentissage fournit un niveau plus élevé de capital humain spécifique que le lycée professionnel. Il procurerait donc alors un avantage aux jeunes embauchés par leur maître d'apprentissage (que nous nommerons par la suite les «stayers ») dans la mesure où ils récupèrent les gains de l'investissement en capital humain spécifique ${ }^{8}$.

- L'apprentissage fournit-il un niveau de capital humain général plus faible ou plus élevé que le lycée professionnel ?

Si le stock de capital humain général est plus faible (respectivement plus élevé) pour les apprentis, les entreprises, toutes choses égales par ailleurs, auront alors tendance à embaucher les jeunes ayant le plus grand stock de capital humain général c'est-à-dire les lycéen(ne)s (respectivement les apprenti(e)s). L'apprentissage peut alors procurer un désavantage (respectivement un avantage) à tous ceux qui ne sont pas employés par leur maître d'apprentissage (que nous appellerons des «movers »), si on suppose que le capital humain acquis par les apprentis est purement spécifique à l'entreprise dans laquelle ils ont effectué leur stage.

Si le stock de capital humain général des apprenti(e)s était plus élevé que celui des lycéen(ne)s, on devrait observer un effet positif de l'apprentissage sur toutes les sorties vers l'emploi (directement depuis le chômage), puisque les lycéens ont moins de capital humain spécifique.

- On peut penser que le choix entre le lycée professionnel et l'apprentissage résulte d'un processus de sélection. Il en est de même pour la distribution entre les «stayers » (apprenti(e)s embauché(e)s par leur maître d'apprentissage) et les «movers » (apprenti(e)s non embauché(e)s par leur maître

8. Pour une analyse détaillée du concept de capital humain voir HASHIMOTO [1981] et Kato [1989]. 
d'apprentissage). D'une part, le fait d'être un «mover» peut donner un effet de signal négatif. Dans ce cas, pour des apprenti(e)s et des lycéen(ne)s ayant un niveau de capital humain général équivalent et des caractéristiques générales observées identiques, les apprenti(e)s non embauché(e)s par leur maître d'apprentissage pourraient être désavantagé(e)s. Inversement, les « movers » pourraient être des apprentis possédant plutôt des caractéristiques inobservables plus positives que la moyenne, et qui choisissent eux-mêmes de partir de l'entreprise ${ }^{9}$, transférant ainsi dans des entreprises plus attirantes (du point de vue des salaires, des conditions de travail, des possibilités de promotion et de carrière...) une partie au moins du capital humain spécifique (au secteur et non plus strictement à l'entreprise) acquis en apprentissage. L'effet global de l'apprentissage est alors indéterminé.

Le modèle considéré nous permet d'une part de corriger le biais de sélection induit par le choix de l'apprentissage ou du lycée professionnel, et de déterminer son sens (apprentissage choisi ou subi). D'autre part, grâce à la prise en compte de phénomène d'embauche chez le maître d'apprentissage, nous pourrons voir si l'effet positif du passage par l'apprentissage sur l'accès au premier emploi s'applique à l'ensemble des apprentis ou uniquement aux « stayers ».

La modélisation considérée va nous permettre d'estimer simultanément - la probabilité de passage par l'apprentissage (la variable associée à l'apprentissage sera notée $Y_{a}$ ),

- la probabilité d'être embauché par son maître d'apprentissage (stayers) pour la population des apprentis (la variable associée à l'embauche par le maître de stage sera notée $Y_{m}$ ),

- les paramètres de la distribution des durées de chômage $Y_{c}$ et par conséquent, étant donnée la modélisation retenue, ceux associés à la probabilité de trouver immédiatement un emploi ${ }^{10}$.

Le modèle statistique détaillé est donné dans l'encadré 1. Les différents cas pouvant être observés dans cette modélisation sont donnés par la figure 1. Ces cas (au nombre de 7) correspondent aux différentes contributions à la vraisemblance données dans l'annexe 2. Il est important de noter que les différentes branches de l'arbre ne correspondent pas ici à des décisions séquentielles. Dans ce travail, notre premier objectif est d'étudier la probabilité individuelle de trouver un emploi (quel que soit le type de cet emploi) à la sortie du système éducatif. Pour les apprenti(e)s, nous nous intéresserons simultanément à la probabilité d'être embauché dans l'entreprise où le jeune a fait son apprentissage. Cela implique nécessairement, d'une part que tout individu travaillant chez son maître de stage est en emploi immédiat et d'autre part que tout individu en chômage n'a pas été embauché par son maître de stage 11. Par conséquent, il serait tout à fait possible, dans la figure 1, d'inverser les nœuds de l'arbre «a trouvé un emploi immédiatement» et «a été

9. Si on suppose que les entreprises qui acceptent des apprentis sont caractérisées par de mauvaises conditions de travail ou des possibilités de carrière limitées par rapport aux autres entreprises du secteur.

10. Comme l'année scolaire se termine à la fin du mois de juin, et que juillet et août sont des périodes de vacances pour la plupart des étudiants, « immédiate » signifie ici dans les deux mois.

11. Cette logique correspond aux observations empiriques : tous les apprentis recrutés par l'entreprise dans laquelle ils ont effectué leur apprentissage l'ont été dans les deux mois qui suivent la fin des études. 


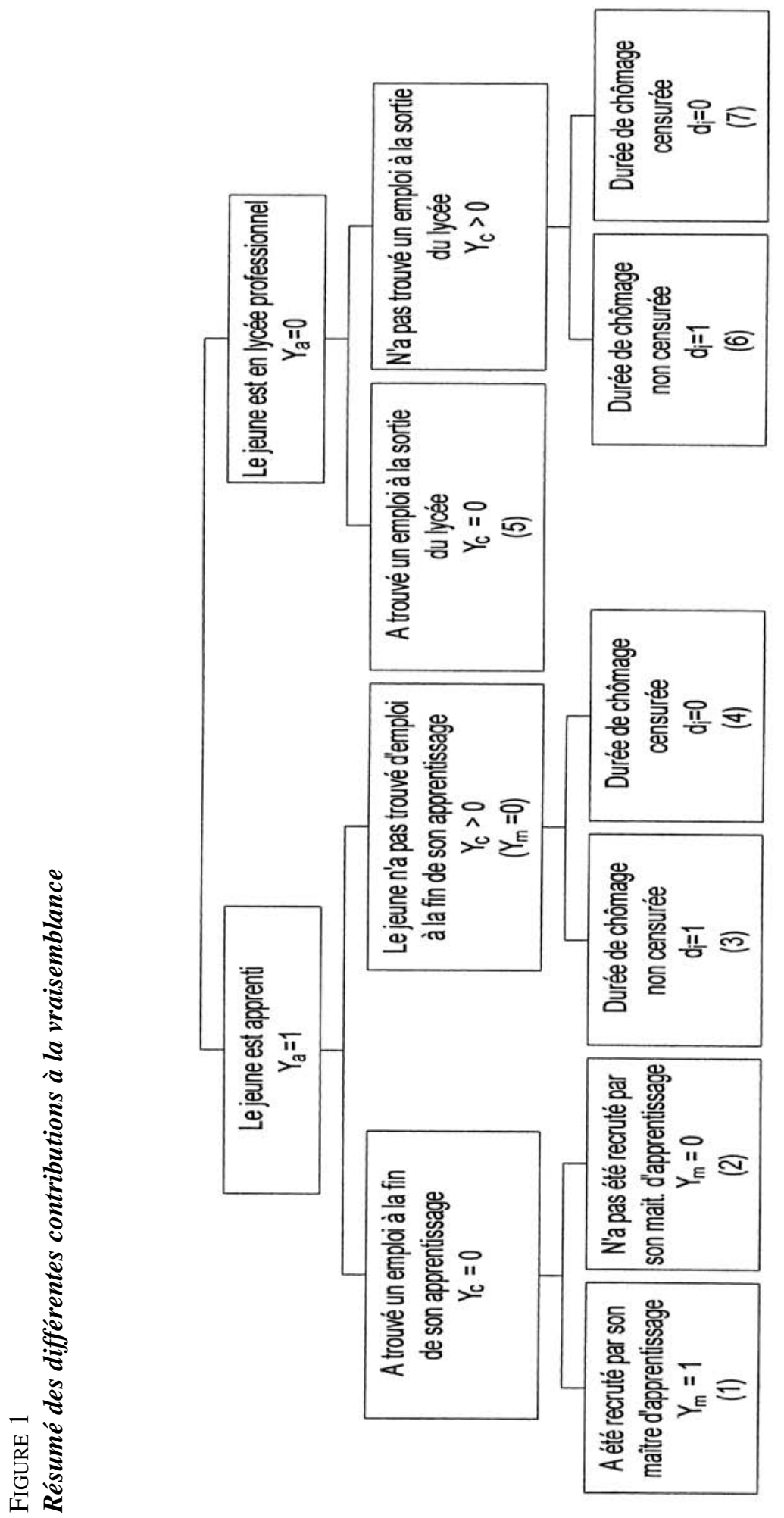




\section{ENCADRÉ 1}

\section{Le modèle}

Soient $Y_{a}$ et $Y_{m}$ des variables dichotomiques aléatoires. Pour un individu, les réalisations de ces variables sont définies par :

$$
\begin{aligned}
& y_{a}=\left\{\begin{array}{l}
1 \text { si l'individu choisit l'apprentissage, } \\
0 \text { sinon } ;
\end{array}\right. \\
& y_{m}=\left\{\begin{array}{l}
1 \text { si l'individu trouve un emploi chez son maître d'apprentissage, } \\
0 \text { sinon. }
\end{array}\right.
\end{aligned}
$$

Soient $Y_{a}^{*}, Y_{m}^{*}$ des variables latentes aléatoires. Pour un individu, la réalisation de ces variables est donnée par

$$
\begin{aligned}
& y_{a}^{*}=X_{a}^{\prime} \beta_{a}+u_{a}, \\
& y_{m}^{*}=X_{m}^{\prime} \beta_{m}+u_{m},
\end{aligned}
$$

où $X_{a}, X_{m}$ sont des vecteurs de régresseurs ; $\beta_{a}, \beta_{m}$ des vecteurs de paramètres et $u_{a}, u_{m}$ sont des réalisations des termes d'erreurs aléatoires $U_{a}, U_{m}$.

La relation (1) peut donc s'écrire

$$
\begin{aligned}
& y_{a}=\left\{\begin{array}{l}
1 \text { si } y_{a}^{*} \geqslant 0, \\
0 \text { sinon } ;
\end{array}\right. \\
& y_{m}=\left\{\begin{array}{l}
1 \text { si } y_{m}^{*} \geqslant 0, \\
0 \text { sinon. }
\end{array}\right.
\end{aligned}
$$

Afin de modéliser la situation vis-à-vis du marché du travail (emploi ou chômage ainsi que la durée de chômage éventuelle) nous définissons la variable suivante :

$$
y_{c}=\ln t_{c}= \begin{cases}\min \left(X_{c}^{\prime} \beta_{c}+y_{a} \beta_{a c}+u_{c}, \ln \tau_{c}\right) & \text { si } y_{c}^{*}=X_{c} \beta_{c}+y_{a} \beta_{a c}+u_{c}>0, \\ 0 & \text { sinon } ;\end{cases}
$$

où $X_{c}$ et $\beta_{c}$ sont respectivement un vecteur de régresseurs et paramètres, $u_{c}$ est la réalisation d'un terme d'erreur aléatoire $U_{c}, t_{c}$ est l'observation de la variable aléatoire associée à la durée de chômage $T_{c}$ et $\tau_{c}$ est la durée censurée. Le modèle de durée considéré est un modèle à vie accélérée. La distribution des durées est supposée log linéaire et $T_{c}$ suit une distribution log normale ${ }^{12}$.

Nous faisons l'hypothèse que les termes d'erreurs $\left(u_{a}, u_{m}, u_{c}\right)$ suivent une loi normale de moyenne zéro et de matrice des variances-covariances $\Sigma$ donnée par

$$
\Sigma=\left(\begin{array}{ccc}
1 & \rho_{a m} & \rho_{a c} \sigma_{c} \\
\rho_{a m} & 1 & \rho_{m c} \sigma_{c} \\
\rho_{a c} \sigma_{c} & \rho_{m c} \sigma_{c} & \sigma_{c}^{2}
\end{array}\right)
$$

Les contributions à la vraisemblance ainsi que la fonction de vraisemblance associées aux deux modèles sont données dans l'annexe 2 .

12. Cette modélisation peut être comparée à un modèle Tobit. Le modèle Tobit dans l'analyse des durées de chômage a été utilisé, par exemple, par FougÈRE et SÉRANDON [1992]), par DoLTON et al. [1994]. 
embauché(e) par son maître d'apprentissage » sans que cela ne modifie les résultats car nous observons exactement les mêmes contributions à la vraisemblance (voir annexe 2). Une modélisation alternative pourrait s'appuyer sur une analyse multivariée. Dans un tel modèle le nombre de situations possibles pour les apprenti(e)s et les lycéen(ne)s serait différent. Les apprenti(e)s pourraient être en situation de chômage ou être soumis à deux types d'offres d'emploi : celles proposées par les entreprises dans lesquelles les jeunes ont effectué leur apprentissage et celle proposées par les autres entreprises. On aurait alors pour ces jeunes trois états : le chômage, l'embauche «chez son maître de stage » et l'embauche «ailleurs ». Les lycéen(ne)s n'auraient que deux alternatives : le chômage ou l'emploi.

Notre objectif étant de privilégier l'approche dichotomique emploi immédiat/chômage qui correspond plutôt à la perspective d'un chômage subi, plus réaliste, nous semble-t-il, pour ce qui concerne cette population peu diplômée, notre modélisation semble bien adaptée. Elle nous permet, de plus, de pouvoir comparer directement pour les apprentis et les lycéens ces deux situations.

\section{Résultats}

\subsection{Les estimations}

Pour les raisons évidentes présentées au paragraphe 2, les estimations ont été menées séparément pour les hommes et pour les femmes avec (AC) et sans (SC) coefficients de corrélation entre les termes d'erreur. Les résultats sont donnés dans les tableaux $3 \mathrm{~A}$ et $3 \mathrm{~B}$.

Intéressons-nous tout d'abord à la probabilité d'être apprenti. La catégorie socio-professionnelle des parents n'a aucun effet sur la probabilité d'être apprenti. Le fait d'avoir obtenu le BEPC (qui est un diplôme du système éducatif général) diminue la probabilité de choisir une formation préparée par apprentissage. Certaines différences régionales apparaissent également. Le lycée professionnel fait parti du système scolaire standard, et son offre est déterminée par des normes (relatives au nombre d'élèves) qui sont approximativement les mêmes dans tout le pays. À l'inverse, l'offre d'apprentissage varie sur une échelle plus grande entre les régions. Aussi, les différences régionales dans le choix de l'apprentissage peuvent être interprétées comme le reflet d'effets d'offre.

Pour cette probabilité, les modèles avec et sans coefficient de corrélation donnent des résultats très similaires. Les travaux réalisés par M. SollogouB et V. ULRICH [1998] montrent que les variables liées au retard scolaire, à la classe de sortie vers la formation professionnelle (classe de cinquième ou classe de troisième) et à la taille de la famille ont un effet significatif sur cette probabilité. Toutefois, étant données les informations disponibles dans l'enquête, il n'est pas possible d'inclure de telles variables dans la modélisation.

Examinons maintenant la probabilité, pour un apprenti, d'être embauché par son maître d'apprentissage. Il apparaît que l'obtention du diplôme augmente, toutes choses égales par ailleurs, cette probabilité. Avoir suivi une formation 


\section{TABLEAU 3A}

\section{Estimations de la probabilité d'être apprenti(e)}

\begin{tabular}{|c|c|c|c|c|}
\hline \multirow[t]{2}{*}{ Variables explicatives } & \multicolumn{2}{|c|}{ Hommes } & \multicolumn{2}{|c|}{ Femmes } \\
\hline & $\mathrm{SC}$ & $\mathrm{AC}$ & $\mathrm{SC}$ & $\mathrm{AC}$ \\
\hline Constante & $\begin{array}{c}0.21 \\
(0.10)\end{array}$ & $\begin{array}{c}0.26 \\
(0.10)\end{array}$ & $\begin{array}{r}-0.24 \\
(0.10)\end{array}$ & $\begin{array}{r}-0.23 \\
(0.09)\end{array}$ \\
\hline Titulaire du BEPC & $\begin{array}{r}-1.03 \\
(0.16)\end{array}$ & $\begin{array}{r}-0.92 \\
(0.17)\end{array}$ & $\begin{array}{r}-0.74 \\
(0.13)\end{array}$ & $\begin{array}{r}-0.73 \\
(0.12)\end{array}$ \\
\hline $\begin{array}{l}\text { Région (réf. régions Parisienne ou Centre) } \\
\text { - Ouest }\end{array}$ & $\begin{array}{c}0.20 \\
(0.15)\end{array}$ & $\begin{array}{c}0.13 \\
(0.13)\end{array}$ & $\begin{array}{c}0.09 \\
(0.12)\end{array}$ & $\begin{array}{c}0.07 \\
(0.12)\end{array}$ \\
\hline - Sud-Sud Ouest & $\begin{array}{c}0.42 \\
(0.31)\end{array}$ & $\begin{array}{c}0.40 \\
(0.25)\end{array}$ & $\begin{array}{c}0.73 \\
(0.25)\end{array}$ & $\begin{array}{c}0.70 \\
(0.22)\end{array}$ \\
\hline - Nord & $\begin{array}{c}-0,36 \\
(0.19)\end{array}$ & $\begin{array}{r}-0.38 \\
(0.18)\end{array}$ & $\begin{array}{l}-0.72 \\
(0.16)\end{array}$ & $\begin{array}{r}-0.74 \\
(0.15)\end{array}$ \\
\hline - Est & $\begin{array}{c}0.22 \\
(0.18)\end{array}$ & $\begin{array}{c}0.29 \\
(0.15)\end{array}$ & $\begin{array}{c}0.66 \\
(0.18)\end{array}$ & $\begin{array}{c}0.66 \\
(0.15)\end{array}$ \\
\hline Qualification du père (réf. Ouvrier ou employé) & & & & \\
\hline - Père travailleur indépendant & $\begin{array}{c}0.04 \\
(0.23)\end{array}$ & $\begin{array}{c}0.17 \\
(0.17)\end{array}$ & $\begin{array}{l}0.243 \\
(0.16)\end{array}$ & $\begin{array}{c}0.22 \\
(0.13)\end{array}$ \\
\hline - Père cadre & $\begin{array}{c}0.17 \\
(0.39)\end{array}$ & $\begin{array}{c}0.15 \\
(0.31)\end{array}$ & $\begin{array}{l}-0.50 \\
(0.33)\end{array}$ & $\begin{array}{l}-0.50 \\
(0.30)\end{array}$ \\
\hline
\end{tabular}

Les chiffres en italique sont significatifs à $10 \%$, ceux en gras sont significatifs à $5 \%$, ceux en gras et en italique sont significatifs à $1 \%$. Les chiffres entre parenthèses sont les écart-types.

\section{TABLEAU 3B}

\section{Probabilité d'être embauché par son maître d'apprentissage}

\begin{tabular}{|l|c|c|c|c|}
\hline Variables explicatives & \multicolumn{2}{|c|}{ Hommes } & \multicolumn{2}{c|}{ Femmes } \\
\hline & SC & AC & SC & AC \\
\hline Constante & -0.5 & 0.13 & 0.18 & $\mathbf{- 0 . 6 0}$ \\
& $(0.23)$ & $(0.22)$ & $(0.21)$ & $\mathbf{( 0 . 2 2 )}$ \\
\hline Titulaire du diplôme (CAP ou BEP) & 0.28 & $\mathbf{0 . 3 0}$ & 0.19 & 0.33 \\
& $(0.24)$ & $\mathbf{( 0 . 1 6 )}$ & $(0.24)$ & $(0.15)$ \\
\hline Spécialité du diplôme* & & & & \\
- Alimentation & $\mathbf{- 0 . 7 5}$ & $\mathbf{- 0 . 6 0}$ & - & - \\
& $(\mathbf{0 . 2 0})$ & $\mathbf{( 0 . 1 4 )}$ & & \\
- Hôtellerie & -0.15 & -0.06 & $-\boldsymbol{0 . 8 7}$ & $\mathbf{- 0 . 6 1}$ \\
& $(0.41)$ & $(0.29)$ & $(\boldsymbol{0 . 2 7})$ & $(\boldsymbol{0 . 2 4})$ \\
- Gestion & -0.31 & -0.29 & -0.22 & -0.08 \\
& $(0.44)$ & $(0.23)$ & $(0.19)$ & $(0.18)$ \\
- Métiers liés à l'esthétique & - & - & -0.16 & -0.05 \\
& & & $(0.24)$ & $(0.20)$ \\
\hline
\end{tabular}

* Les spécialités de référence sont : électricité, mécanique et bâtiment ainsi que les autres spécialités pour la population des hommes et les métiers de la confection ainsi que les autres spécialités pour la population des femmes.

Les chiffres en italique sont significatifs à $10 \%$, ceux en gras sont significatifs à $5 \%$, ceux en gras et en italique sont significatifs à $1 \%$. Les chiffres entre parenthèses sont les écart-types. 
dans le secteur alimentaire pour les hommes, et dans le secteur hôtelier pour les femmes, apparaît comme significativement négatif. Pour la population des hommes, le modèle avec coefficient de corrélation montre qu'il existe une relation négative entre la probabilité de choisir l'apprentissage et celle d'être embauché à la sortie du système éducatif dans l'entreprise où a été réalisé l'apprentissage. Les caractéristiques individuelles non observables favorisant l'accès à l'apprentissage semblent être défavorables à l'embauche de cet individu. Il existerait donc un biais de sélection négatif contre les apprentis, les entreprises choisissant principalement de garder les apprentis qui ne présentent pas les caractéristiques négatives correspondant au biais de sélection ${ }^{13}$.

\section{TABLEAU 3C}

\section{Durée de chômage et probabilité d'emploi immédiat}

\begin{tabular}{|c|c|c|c|c|}
\hline \multirow[t]{2}{*}{ Variables explicatives $^{14}$} & \multicolumn{2}{|c|}{ Hommes } & \multicolumn{2}{|c|}{ Femmes } \\
\hline & $\mathrm{SC}$ & $\mathrm{AC}$ & SC & $\mathrm{AC}$ \\
\hline Constante & $\begin{array}{r}-0.64 \\
(0.66)\end{array}$ & $\begin{array}{c}1.60 \\
(0.98)\end{array}$ & $\begin{array}{c}0.89 \\
(0.32)\end{array}$ & $\begin{array}{c}1.04 \\
(0.25)\end{array}$ \\
\hline Titulaire du diplôme préparé & $\begin{array}{r}-1.65 \\
(0.53)\end{array}$ & $\begin{array}{r}-1.70 \\
(0.55)\end{array}$ & $\begin{array}{r}-0.88 \\
(0.31)\end{array}$ & $\begin{array}{r}-0.87 \\
(0.24)\end{array}$ \\
\hline Diplôme préparé dans le secteur tertiaire & $\begin{array}{c}0.92 \\
(0.50)\end{array}$ & $\begin{array}{c}0.67 \\
(0.50)\end{array}$ & $\begin{array}{c}-0.31 \\
(0.29)\end{array}$ & $\begin{array}{r}-0.40 \\
(0.70)\end{array}$ \\
\hline Diplôme préparé par apprentissage & $\begin{array}{r}-1.79 \\
(0.48)\end{array}$ & $\begin{array}{r}-5.74 \\
(1.62)\end{array}$ & $\begin{array}{r}-0.59 \\
(0.28)\end{array}$ & $\begin{array}{r}-0.94 \\
(0.40)\end{array}$ \\
\hline Mère travaille & - & - & $\begin{array}{l}-0.37 \\
(0.30)\end{array}$ & $\begin{array}{l}-0.41 \\
(0.22)\end{array}$ \\
\hline Père travaille & $\begin{array}{r}-1.09 \\
(0.54)\end{array}$ & $\begin{array}{r}-1.03 \\
(0.70)\end{array}$ & - & - \\
\hline $\begin{array}{l}\text { CSP du père (réf. Ouvrier ou employé) } \\
\text { - Père travailleur indépendant } \\
\text { - Père cadre }\end{array}$ & $\begin{array}{c}0.85 \\
(0.72) \\
0.47 \\
(1.28)\end{array}$ & $\begin{array}{c}\mathbf{1 . 5 9} \\
(\mathbf{0 . 8 3}) \\
0.50 \\
(1.55)\end{array}$ & $\begin{array}{r}-0.47 \\
(0.39) \\
-1.32 \\
(1.04)\end{array}$ & $\begin{array}{r}-0.36 \\
(0.45) \\
-\mathbf{1 . 2 9} \\
(\mathbf{0 . 6 4 )}\end{array}$ \\
\hline$\sigma_{c}$ & $\begin{array}{c}4.05 \\
(0.40)\end{array}$ & $\begin{array}{c}4.47 \\
(0.52)\end{array}$ & $\begin{array}{c}2.70 \\
(0.19)\end{array}$ & $\begin{array}{c}2.70 \\
(0.17)\end{array}$ \\
\hline$\delta_{12}$ & - & $\begin{array}{r}-0.80 \\
(0.42)\end{array}$ & - & $\begin{array}{c}0.06 \\
(0.13)\end{array}$ \\
\hline$\delta_{13}$ & - & $\begin{array}{c}0.70 \\
(0.27)\end{array}$ & - & $\begin{array}{c}0.10 \\
(0.92)\end{array}$ \\
\hline$\delta_{23}$ & - & $\begin{array}{r}-2.61 \\
(3.93)\end{array}$ & - & $\begin{array}{r}-2.68 \\
(5.54)\end{array}$ \\
\hline
\end{tabular}

Les chiffres en italique sont significatifs à $10 \%$, ceux en gras sont significatifs à $5 \%$, ceux en gras et en italique sont significatifs à $1 \%$. Les chiffres entre parenthèses sont les écart-types.

13. Une interprétation alternative serait que les apprentis ont des caractéristiques favorables, corrélées positivement avec le fait de choisir de ne pas rester dans son entreprise d'apprentissage (aux caractéristiques supposées plutôt négatives dans ce cas, $c f$. section 3). Mais cette interprétation est en contradiction avec les résultats ci-dessous concernant la durée du chômage.

14. Afin de contraindre les corrélations à être comprises entre -1 et 1 , la covariance entre les termes d'erreurs $u_{i}$ et $u_{j}, \rho_{i j}=\tanh \left(\delta_{i j}\right)$. 
Avant de s'intéresser aux caractéristiques individuelles ayant un effet sur la probabilité d'être en situation d'emploi à la sortie du système éducatif (tableau $3 \mathrm{C}$ ), on peut noter que la corrélation entre les termes d'erreur associés à l'embauche dans l'entreprise où le jeune a réalisé son apprentissage et le chômage est négative (et très proche de -1 ). Les caractéristiques individuelles non observables favorisant l'embauche chez le maître d'apprentissage semblent donc être favorable à l'embauche dans une autre entreprise ${ }^{15}$.

Les principales variables qui affectent significativement la probabilité d'obtenir un emploi immédiat après la sortie du système éducatif (ou de façon équivalente la durée de chômage) sont en fait le type de formation (apprentissage ou lycée professionnel) et l'obtention du diplôme.

Les apprenti(e)s ont de meilleures chances d'obtenir immédiatement un emploi que les lycéen(ne)s. Cet impact est particulièrement élevé pour la population masculine. Cet effet est encore renforcé lorsque les corrélations sont prises en compte. Le modèle avec corrélation montre une relation positive et significative entre le terme d'erreur associé à la probabilité d'être apprenti et celui associé à la durée de chômage. Ce résultat n'est vrai que pour la population masculine. Cela signifie que, toutes choses égales par ailleurs, les jeunes hommes ayant une probabilité relativement forte de devenir apprentis sont aussi ceux qui ont une probabilité relativement faible de trouver un emploi immédiatement après la sortie du système éducatif ou, de manière équivalente, ont une durée moyenne de chômage relativement plus longue. La population des apprentis semble donc être défavorisée par rapport à celle des lycéens quant à l'accès direct sur le marché de l'emploi. Toutefois, le coefficient associé à la variable caractérisant le passage par l'apprentissage est négatif, significatif et relativement élevé. Il semblerait donc que les individus ayant tendance à préparer leur diplôme par apprentissage aient moins de chance de trouver immédiatement un emploi mais, le passage par l'apprentissage contrebalance cet effet négatif. L'apprentissage apparaît alors, pour cette population, comme très bénéfique à ces jeunes hommes. Quel que soit le sexe considéré, l'obtention du diplôme exerce également un effet important sur la probabilité de trouver immédiatement un emploi ${ }^{16}$. Dans une moindre mesure et pour la population masculine seulement, le fait d'avoir un père en emploi augmente la probabilité de trouver un emploi immédiatement. À l'inverse, le fait d'avoir suivi une formation tertiaire et plus encore d'avoir un père travailleur indépendant a tendance à augmenter la durée moyenne de chômage. Il apparaît donc que les apprenti(e)s (a fortiori les diplômé(e)s) ont, toutes choses égales par ailleurs, une probabilité plus forte de trouver un emploi immédiatement après la sortie du système éducatif. Des études portant sur les durées de chômage montrent que, conditionnellement au fait d'être chômeurs, les durées moyennes de chômage des apprentis sont, en particulier pour les hommes, plus élevées que celles des lycéens. Ils sembleraient donc que, si les apprentis ne trouvent pas directement un emploi, ils ont plus de mal que les lycéens à s'insérer ${ }^{17}$. La modélisation retenue ici ne nous permet pas d'aboutir à une telle

\footnotetext{
15. La présence d'hétérogénéité supplémentaire (en particulier de variables continues) permettrait peut-être de rendre moins proche de -1 ce coefficient. Malheureusement, l'enquête ne nous fournit pas d'autre information que celle utilisée.

16. L'étude réalisée par BONNAL, FLEURY et RoCHARD [1999] sur l'insertion à court terme des apprentis et des lycéens met également en évidence ce résultat.

17. Ce résultat se vérifie par exemple dans Bonnal et MENDEs [2000].
} 
conclusion. Pour l'obtenir, il aurait été nécessaire d'estimer un modèle plus complexe de type Tobit généralisé associé à la durée de chômage.

\subsection{Calculs et simulations de probabilités}

À partir de ces estimations, il est possible de calculer, selon des caractéristiques individuelles particulières, les probabilités d'être apprenti, d'être en emploi à la sortie du système éducatif ou encore la probabilité d'être embauché par son maître d'apprentissage. Étant donné que le type de formation et l'obtention du diplôme à la suite de cette formation jouent un rôle important pour l'accès immédiat à l'emploi, nous nous sommes essentiellement intéressés à ces deux caractéristiques ${ }^{18}$. Les probabilités estimées tout d'abord dans ce travail (tableaux 4A et 4B) sont issues du modèle avec corrélation qui est préféré pour les deux populations lorsque l'on réalise un test du quotient de vraisemblance par exemple. Toutes ces probabilités sont calculées conditionnellement au type de formation (apprenti(e) - lycéen(ne)), ces probabilités ayant elles-mêmes été calculées à la moyenne des régresseurs.

TABLEAU 4A

Probabilité d'être en emploi à la sortie du système éducatif 19

\begin{tabular}{|l|c|c|c|c|}
\hline & Apprenti & Lycéen & Apprentie & Lycéenne \\
\hline \multirow{2}{*}{ - diplômé(e) } & 86.1 & 74.1 & 66.1 & 58.4 \\
& $(0.02)$ & $(0.03)$ & $(0.03)$ & $(0.04)$ \\
- non diplômé(e) & 75.1 & 59.5 & 53.6 & 45.6 \\
& $(0.04)$ & $(0.04)$ & $(0.04)$ & $(0.05)$ \\
\hline
\end{tabular}

TABLEAU 4B

Probabilité d'être en emploi pour la population des apprenti(e)s, à la sortie du système éducatif

\begin{tabular}{|l|c|c|}
\hline & Apprenti & Apprentie \\
\hline $\begin{array}{l}\text { Probabilité d'être en emploi sachant que le jeune } \\
\text { n'a pas été embauché par son maître d'apprentissage }\end{array}$ & & \\
- diplômé(e) & 75.7 & 47.0 \\
& $(0.04)$ & $(0.04)$ \\
- non diplômé(e) & 64.1 & 38.6 \\
& $(0.05)$ & $(0.05)$ \\
\hline Probabilité de ne pas être embauché par son maître & & \\
d'apprentissage sachant que le jeune a trouvé un emploi & & \\
- diplômé(e) & 52.7 & 51.3 \\
& $(0.04)$ & $(0.04)$ \\
- non diplômé(e) & 62.0 & 54.4 \\
& $(0.06)$ & $(0.07)$ \\
\hline
\end{tabular}

Les valeurs données entre parenthèses sont les écart-types.

18. Pour le calcul de ces probabilités, les autres variables ont été considérées à la moyenne des régresseurs.

19. Les probabilités sont données en pourcentage, les chiffres entre parenthèses sont les écart-types associés à ces probabilités. 
D'après le tableau 4A, il semblerait que l'apprentissage facilite très sensiblement un accès immédiat à l'emploi : ce type de formation permet une meilleure performance que le lycée professionnel, particulièrement pour les hommes et après correction du biais de sélection dans le choix de l'apprentissage.

Il est également intéressant de comparer les résultats des apprentis qui ne restent pas chez leur maître avec ceux des lycéens : les premiers ne bénéficient pas de capital humain spécifique ${ }^{20}$ et sont, par conséquent, de ce point de vue, dans la même situation que les lycéens. Comme on peut le constater dans le tableau $4 \mathrm{~B}$, les résultats ne sont pas identiques pour les hommes et pour les femmes. Dans le cas masculin, les apprentis diplômés ont la même probabilité que les lycéens de trouver un emploi (75\%). En revanche, pour les non diplômés, il semblerait que les apprentis conservent un avantage dans l'obtention d'un emploi immédiat (64\% des «movers » contre $59.5 \%$ des lycéens trouvent un emploi immédiatement). Dans le cas des hommes, les entreprises ne semblent pas considérer que le lycée professionnel délivre une meilleure qualité ou quantité de capital humain général que l'apprentissage. La situation opposée apparaît chez les femmes, pour lesquelles, mêmes si dans l'ensemble les apprenties réussissent mieux, les «movers » font moins bien $(47 \%$ des « movers », contre $58.4 \%$ des lycéennes diplômées et $38.6 \%$ des «movers » contre $45.6 \%$ des lycéennes non diplômées, trouvent un emploi immédiatement). Cette différence entre les hommes et les femmes est difficile à comprendre, dans la mesure où les cours théoriques suivis par chacun sont les mêmes, aussi bien pour les apprentis que pour les lycéens. Peut-être que la formation des apprenties dans les spécialités féminines (beauté, coiffure, hôtellerie, secrétariat) est plus spécifique que dans les spécialités masculines, ou alors que le biais de sélection négatif et/ou le signal parmi les «movers » est plus important dans cette population.

Pour tenter de mesurer l'efficacité relative des deux types de formation considérées, nous avons calculé la probabilité de sortie immédiate pour un individu aux caractéristiques moyennes à partir des modèles 1 et 2 (tableau 4C).

Pour un jeune quelconque (moyen) nous calculons sa probabilité d'être en emploi après un apprentissage (ou après le lycée) en ayant neutralisé le poids des différences dans les variables observées et non observées qui font qu'il a choisi plutôt l'apprentissage ou plutôt le lycée. Cela consiste à supposer que ce jeune (moyen) a choisi l'apprentissage totalement au hasard, et les probabilités ainsi calculées sont indépendantes des caractéristiques individuelles. Cette méthode ne correspond pas à un calcul rigoureux de probabilités, mais correspond plutôt à une simulation, qui vise à mettre en évidence l'effet « pur» du passage par l'apprentissage. En effet, le modèle 2 corrige le biais de sélection initial pour les apprentis et la différence avec le modèle 1 peut être interprétée comme la mesure du biais de sélection.

Nous avons également calculé la probabilité de sortie immédiate d'un individu aux caractéristiques moyennes passé par l'apprentissage et n'étant pas resté chez son maître d'apprentissage. L'idée, ici, est d'isoler l'effet du capital humain spécifique (à l'entreprise d'apprentissage) et de comparer l'accès à l'emploi d'un apprenti qui n'a pas accumulé de capital humain spécifique avec celui d'un lycéen.

20. Ils pourraient bénéficier de capital humain «sectoriel spécifique », mais alors les lycéens bénéficient aussi d'une certaine formation «spécifique ». 
TABLEAU 4C

Efficacité comparée de l'apprentissage et du lycée professionnel

\begin{tabular}{|l|c|c|c|c|c|c|}
\hline & \multicolumn{3}{|c|}{ Hommes } & \multicolumn{3}{c|}{ Femmes } \\
\hline $\begin{array}{l}\text { Probabilité de sortie } \\
\text { immédiate pour }\end{array}$ & $\begin{array}{c}\text { valeurs } \\
\text { empiriques }\end{array}$ & $\begin{array}{c}\text { modèle 1 } \\
\text { (SC) }\end{array}$ & $\begin{array}{c}\text { modèle 2 } \\
(\mathrm{AC})\end{array}$ & $\begin{array}{c}\text { valeurs } \\
\text { empiriques }\end{array}$ & $\begin{array}{c}\text { modèle 1 } \\
\text { (SC) }\end{array}$ & $\begin{array}{c}\text { modèle 2 } \\
\text { (AC) }\end{array}$ \\
\hline Un apprenti & 82.4 & 82.33 & 88.79 & 66.8 & 62.54 & 88.64 \\
Un lycéen & 67.8 & 68.5 & 47.11 & 53.1 & 53.08 & 79.54 \\
Un apprenti « mover » & 71.28 & 71.6 & 47.88 & 51.03 & 44.71 & 83.25 \\
\hline
\end{tabular}

La correction des différences dans les valeurs moyennes des variables observées fait apparaître peu d'écarts, les fréquences observées étant proches des probabilités calculées dans le modèle sans corrélation (modèle 1).

La correction des différences dans les variables inobservées (modèle 2 contre modèle 1) fait apparaître des différences plus marquées. Ainsi, après prise en compte du biais de sélection, les avantages de l'apprentissage par rapport au lycée professionnel s'accroissent encore pour les deux sexes, avec un accroissement particulièrement élevé pour les femmes.

En isolant l'effet de capital humain spécifique, on constate des effets différenciés chez les hommes et les femmes. Pour les hommes, les apprentis qui ne restent pas chez leur maître d'apprentissage ont toujours une position plus favorable que les lycéens quant à l'accès à l'emploi, la correction du biais de sélection ayant pour effet d'accentuer cet écart. Pour les femmes, l'écart observé en faveur des lycéennes s'accroît avec le modèle 1 mais s'inverse en faveur des apprenties après correction du biais de sélection. Ce dernier résultat doit cependant être considéré avec précaution, dans la mesure où le coefficient de corrélation (entre le terme d'erreur associé à la probabilité d'être apprenti et celui associé à la durée de chômage) n'est pas significatif pour les femmes.

Les entreprises ne semblent donc pas considérer que la formation en apprentissage délivre un enseignement général de moindre qualité que le lycée professionnel.

Les résultats font donc apparaître un effet positif de l'apprentissage sur la probabilité d'accès immédiat à l'emploi, qu'il y ait ou non embauche chez le maître d'apprentissage, cet effet faisant plus que compenser, pour les femmes comme pour les hommes, des caractéristiques a priori défavorables de la population des apprentis.

\section{Conclusion}

Globalement, les apprentis réussissent mieux que les lycéens, l'écart étant particulièrement important pour les hommes. L'effet positif de l'apprentissage résulte principalement des meilleures performances des apprentis en tout début de période : ils sont souvent embauchés (immédiatement ou dans les 
deux mois) dans leur entreprise d'apprentissage. Concernant les hommes ayant suivi un CAP, même quand les apprentis sont des « movers », ils réussissent mieux que les lycéens. Ces résultats sont renforcés lorsque l'on corrige le biais de sélection négatif dans le choix de l'apprentissage. En ce qui concerne les femmes, les apprenties ont un avantage plus faible, et il n'y a pas de biais de sélection significatif. Les résultats montrent donc que l'acquisition de capital humain est valorisante, mais également, pour les hommes, que les entreprises valorisent plus le capital humain acquis par les apprentis que par les lycéens, même quand les rendements de capital humain spécifique à l'entreprise sont supprimés par la sortie de l'entreprise d'apprentissage. Il est également bon de noter que la variable qui a le plus fort effet positif sur la transition de l'école à l'emploi est l'obtention du diplôme, diplôme qui aide non seulement à obtenir un emploi rapidement, mais également un emploi de meilleure qualité ${ }^{21}$. La limite principale à cette conclusion sur les bénéfices de l'apprentissage concerne ce qui se passe par la suite sur le marché du travail : nous avons étudié uniquement l'accès au premier emploi. D'autres études (SOLLOGOUB et UlRICH [1998]) montrent que ces bénéfices semblent se dissiper en partie après quelque temps passé sur le marché du travail.

21. Dans une autre étude, Bonnal, Mendes et Sofer [1998], nous montrons également, avec une approche simplifiée, que les apprentis(e)s ont des emplois plus stables. 


\section{- Références bibliographiques}

AdDison J.T., SIEBERT W.S. (1994). - «Vocational training and the European Community », Oxford Economic Papers, 46(4).

Bonnal L., Fleury L., Rochard M.B. (1999). - «L'insertion professionnelle des apprentis et des lycéens professionnels : des emplois proches des formations suivies », Économie et Statistiques, 323(3), 3-30.

Bonnal L., Mendes S., Sofer C. (1998). - «Access to the first job : a comparison between apprenticeship and vocational school in France», Document de recherche, LEO, 1998-20.

Bonnal L., Mendes S., Sofer C. (2002). - « School-to-work transition : apprenticeship versus vocational school in France », International Journal of Manpower, vol. 23, n 5 , 426-442.

Bonnal L., Mendes S. (2000). - «Faut-il développer la formation par apprentissage ? ", Efficacité versus équité en économie sociale, tome 2, p. 25-36, 499 pages, éditions L'Harmattan.

Bоотн A.L., Satchell S.E. (1994). - «Apprenticeships and job tenure », Oxford Economic Papers, 46(4).

Dolton P.J., Makepeace G.H., Treble J.G. (1994). - « The youth training scheme and the school-to-work transition », Oxford Economic Papers, 46(4), 629-657.

Fougere D., SERANdon A. (1992). - « La transition du système éducatif à l'emploi en France : le rôle des variables scolaires et sociales », Revue d'économie Sociale, $\mathrm{n}^{\circ} 27$ et 28 , Tome 2, p. 89-101.

Hаsнiмото M. (1981). - «Firm-specific human capital as a shared investment», American Economic Review, June, 475-482.

Kато T. (1989). - «Specific and general training in the theory of labor turnover », Economics Letters, 30, 259-262.

Liaisons sociales : «Insertion professionnelle des jeunes. Les stages les contrats », Juillet $1990, \mathrm{n}^{\circ} 10746$.

Liaisons sociales : «Les contrats d'apprentissage, la taxe d'apprentissage », Juin 1993, $\mathrm{n}^{\circ} 11469$.

LYNCH L.M. (1983). - « Job search and youth unemployment », Oxford Economic Papers, 35, 271-282.

MansuY M. (1996). - «La gamme des emplois après un CAP ou un BEP », Éducation et Formation, 45, 115-22.

RYAN P. (2001). - « The school-to-work transition : a cross-national perspective », Journal of Economic Literature, vol. XXXIX, 34-92.

SteEDMAN H. (1993). - «The economics of youth training in Germany », Economic Journal, vol. 103 (420), 1279-1291.

SOllogoub M., UlRich V. (1999). - « Les jeunes en apprentissage ou en lycée professionnel », Économie et Statistiques, 323(3), 31-49.

Torelli N., Trivellato U. (1989). - « Youth unemployment duration from the Italian labour force survey », European economic review, 33, 407-415.

Vialla A. (1997). - « Apprentissage: ruptures, enchaînements de contrats et accès à l'emploi », Note d'Information, 97(22), Ministère de l'Éducation Nationale.

WERQUIN P. (1997). - «1986-1996 : dix ans d'intervention publique sur le marché du travail des jeunes », Économie et Statistique, 304-305(4/5), 121-136.

WERQUIN P. (1996). - «Les dispositifs d'aide à l'insertion des jeunes: différer l'âge d'accès à l'emploi ? », Céreq-Bref, $\mathrm{n}^{\circ} 119$, avril.

WILLIS R.J. (1986). - « Wage determinants: a survey and reinterpretation of human capital earnings functions », Handbook of Labor Economics, Éditeurs Ashenfelter, O et Layard, R, Elsevier Science Publishers BV.

Willis RJ, Rosen S. (1979). - «Education and self-selection», Journal of Political Economy, 87, 7-36. 


\section{Statistiques descriptives complémentaires}

\begin{tabular}{|l|c|c|c|c|c|c|}
\hline & \multicolumn{3}{|c|}{ Hommes } & \multicolumn{3}{c|}{ Femmes } \\
\hline Variables explicatives & Ensemble H & Apprenti & Lycéen & Ensemble F & Apprentie & Lycéenne \\
\hline Situation & & & & & & \\
professionnelle du père & & & & & & \\
$\quad$ - en emploi & 72.8 & 76.4 & 68.1 & 70.6 & 77.3 & 66.9 \\
- au chômage & 20.4 & 17.6 & 24.1 & 21.1 & 17.9 & 22.8 \\
- inactif & 7.8 & 6.0 & 7.8 & 8.3 & 4.8 & 10.3 \\
CSP du père & & & & & & \\
- cadre & 2.8 & 3.2 & 2.3 & 4.7 & 2.7 & 5.9 \\
- employé ou ouvrier & 82.4 & 80.8 & 84.4 & 80.0 & 78.4 & 80.9 \\
- travailleur & & & & & & \\
$\quad$ indépendant & 14.8 & 16.0 & 13.3 & 15.3 & 18.9 & 13.2 \\
\hline Situation professionnelle & & & & & & \\
de la mère & & & & & & \\
- en emploi & 42.5 & 43.9 & 40.7 & 44.6 & 42.9 & 45.6 \\
- au chômage & 29.7 & 33.2 & 25.0 & 29.9 & 35.5 & 26.7 \\
- inactive & 27.8 & 22.9 & 34.3 & 25.5 & 21.6 & 27.7 \\
\hline Région & & & & & & \\
- Est & 15.6 & 17.9 & 12.5 & 15.1 & 24.9 & 9.5 \\
- Sud & 14.2 & 13.9 & 14.7 & 12.3 & 7.6 & 15.0 \\
- Sud Ouest & 5.2 & 6.6 & 3.4 & 3.8 & 6.7 & 2.1 \\
- Ouest & 31.1 & 32.6 & 29.1 & 29.4 & 30.3 & 28.9 \\
- Centre & 13.6 & 13.2 & 14.3 & 15.2 & 18.6 & 13.2 \\
- Nord & 14.2 & 9.9 & 19.8 & 19.2 & 7.0 & 26.1 \\
- Ile de France & 6.1 & 5.9 & 6.2 & 5.0 & 4.9 & 5.2 \\
\hline
\end{tabular}

\section{ANNEXE 2}

\section{Contributions à la vraisemblance}

$l_{1}=\operatorname{Pr}\left(Y_{a}=1, Y_{m}=1, Y_{c}=0\right)=\operatorname{Pr}\left(Y_{c}=0 \mid Y_{a}=1, Y_{m}=1\right) \operatorname{Pr}\left(Y_{a}=1, Y_{m}=1\right)$.

Or, $\operatorname{Pr}\left(Y_{c}=0 \mid Y_{a}=1, Y_{m}=1\right)=1$, en effet, sachant que l'on est apprenti et que l'on est embauché par son maître de stage, la probabilité d'être en emploi est égale à 1 . Par conséquent

$$
l_{1}=\operatorname{Pr}\left(Y_{a}=1, Y_{m}=1\right)=\int_{-X_{a}^{\prime} \beta_{a}}^{+\infty} \int_{-X_{m}^{\prime} \beta_{m}}^{+\infty} f_{a m}\left(u_{a}, u_{m}\right) d u_{m} d u_{a}
$$




$$
\begin{aligned}
l_{2}= & \operatorname{Pr}\left(Y_{a}=1, Y_{m}=0, Y_{c}=0\right) \\
& =\int_{-X_{a}^{\prime} \beta_{a}}^{+\infty} \int_{-\infty}^{-X_{m}^{\prime} \beta_{m}} \int_{-\infty}^{-X_{c}^{\prime} \beta_{c} / \sigma_{c}} f_{a m c}\left(u_{a}, u_{m}, u_{c}\right) d u_{c} d u_{m} d u_{a},
\end{aligned}
$$

En ce qui concerne les apprentis au chômage on a, a priori, deux contributions (sans tenir compte de la censure).

Soit : $\operatorname{Pr}\left(Y_{a}=1, Y_{m}=1, Y_{c}>0\right)=\operatorname{Pr}\left(Y_{m}=1 \mid Y_{a}=1, Y_{c}>0\right) \operatorname{Pr}\left(Y_{a}=1, Y_{c}>0\right)$,

Or, $\operatorname{Pr}\left(Y_{m}=1 \mid Y_{a}=1, Y_{c}>0\right)=0$. Par conséquent cette contribution n'apparaît pas dans l'analyse. En effet, on ne peut pas, d'après la définition des variables, être simultanément embauché par son maître de stage et en situation de chômage.

Soit : $\operatorname{Pr}\left(Y_{a}=1, Y_{m}=0, Y_{c}>0\right)=\operatorname{Pr}\left(Y_{m}=0 \mid Y_{a}=1, Y_{c}>0\right) \operatorname{Pr}\left(Y_{a}=1, Y_{c}>0\right)$

Or, $\operatorname{Pr}\left(Y_{m}=0 \mid Y_{a}=1, Y_{c}>0\right)=1$. En effet, lorsque le jeune est en situation de chômage, la probabilité qu'il ne soit pas embauché chez son maître de stage est égale à 1 . Par conséquent

$$
\operatorname{Pr}\left(Y_{a}=1, Y_{m}=0, Y_{c}>0\right)=\operatorname{Pr}\left(Y_{m}=0 \mid Y_{a}=1, Y_{c}>0\right) \operatorname{Pr}\left(Y_{a}=1, Y_{c}>0\right)
$$

et on peut en déduire les contributions $l_{3}$ (la durée de chômage observée n'est pas censurée) et $\ell_{4}$ (la durée de chômage observée est censurée).

$$
\begin{aligned}
& l_{3}=\operatorname{Pr}\left(Y_{a}=1, y_{c}^{*}=y_{c} \mid d=1\right) \\
& =\operatorname{Pr}\left(Y_{a}=1 \mid y_{c}^{*}=y_{c}, d=1\right)^{*} \operatorname{Pr}\left(y_{c}^{*}=y_{c} \mid d=1\right) \\
& =\int_{-X_{a}^{\prime} \beta_{a}}^{+\infty} f_{a \mid c}\left(u_{a} \mid u_{c}=\ln t_{c}-X_{c}^{\prime} \beta_{c}-y_{a} \beta_{a c}\right) d u_{c} \\
& * \frac{1}{\sigma_{c}} \phi\left(\frac{\ln t_{c}-X_{c}^{\prime} \beta_{c}-y_{a} \beta_{a c}}{\sigma_{c}}\right) \\
& l_{4}=\operatorname{Pr}\left(Y_{a}=1, y_{c}^{*}=y_{c} \mid d=0\right) \\
& =\int_{-X_{a}^{\prime} \beta_{a}}^{+\infty} \int_{\left(\ln t_{c}-X_{c}^{\prime} \beta_{c}-y_{a} \beta_{a c}\right) / \sigma_{c}}^{+\infty} f_{a c}\left(u_{a}, u c\right) d u_{c} d u_{a}, \\
& l_{5}=\operatorname{Pr}\left(Y_{a}=0, Y_{c}=0\right)=\int_{-\infty}^{-X_{a}^{\prime} \beta_{a}} \int_{-\infty}^{-X_{c}^{\prime} \beta_{c} / \sigma_{c}} f_{a c}\left(u_{a}, u_{c}\right) d u_{c} d u_{a}, \\
& l_{6}=\operatorname{Pr}\left(Y_{a}=0, Y_{e}=0, Y_{c}=y_{c}, d=1\right) \\
& =\operatorname{Pr}\left(Y_{a}=0 \mid y_{c}^{*}=y_{c}, d=1\right) \operatorname{Pr}\left(y_{c}^{*}=y_{c} \mid d=1\right) \\
& =\int_{-\infty}^{-X_{a}^{\prime} \beta_{a}} f_{a \mid c}\left(u_{a} \mid u_{c}=\ln t_{c}-X_{c}^{\prime} \beta_{c}\right) d u_{a} \frac{1}{\sigma_{c}} \varphi_{c}\left(\frac{\ln t_{c}-X_{c}^{\prime} \beta_{c}}{\sigma_{c}}\right) \text {, }
\end{aligned}
$$




$$
\begin{aligned}
l_{7}= & \operatorname{Pr}\left(Y_{a}=0, y_{c}^{*}=y_{c} \mid d=1\right) \\
= & \operatorname{Pr}\left(Y_{a}=0 \mid y_{c}^{*}=y_{c}, d=1\right) \operatorname{Pr}\left(y_{c}^{*}=y_{c} \mid d=1\right) \\
= & \int_{-\infty}^{-X_{a} \beta_{a}} \int_{\left(\ln t_{c}-X_{c}^{\prime} \beta_{c}-y_{a} \beta\right) / \sigma_{c}}^{+\infty} f_{a c}\left(u_{a}, u_{c}\right) d u_{c} d u_{a} .
\end{aligned}
$$

$\varphi$ est la fonction de densité d'une distribution normale univariée centrée et réduite.

La contribution à la vraisemblance de l'individu $i$ s'écrit alors :

$$
\begin{aligned}
L_{i}\left(\beta_{a}, \beta_{m}, \beta_{c}, \Sigma\right) & \\
= & l_{1}^{y_{a} y_{e} y_{m}} l_{2}^{y_{a} y_{e}\left(1-y_{m}\right)} l_{3}^{y_{a}\left(1-y_{e}\right) d} \\
& l_{4}^{y_{a}\left(1-y_{e}\right)(1-d)} l_{5}^{\left(1-y_{a}\right) y_{e}} l_{6}^{\left(1-y_{a}\right)\left(1-y_{e}\right) d} l_{7}^{\left(1-y_{a}\right)\left(1-y_{e}\right)(1-d)}
\end{aligned}
$$

où $d$ est l'indicateur de censure prenant la valeur 1 si l'individu $i$ est embauché à la fin de l'épisode observé de chômage (la durée de chômage est alors non censurée) et 0 sinon. $y_{e}$ prend la valeur 1 si l'individu $i$ a trouvé un emploi immédiatement après la sortie du système éducatif et la valeur 0 sinon.

Étant donné que $\left.\rho_{u v} \in\right]-1,1[$ nous supposons que

$$
\rho_{u v}=\tanh \left(\delta_{u v}\right)=\frac{\exp \left(\delta_{u v}\right)-\exp \left(-\delta_{u v}\right)}{\exp \left(\delta_{u v}\right)+\exp \left(-\delta_{u v}\right)}, \delta_{u v} \in R
$$

